\title{
Medical image segmentation algorithm based on multi-scale color wavelet texture
}

\author{
Qi Zhang*, Yan Li, \\ College of Finance and Information, Ningbo University of Finance and Economics, \\ Ningbo, 315175 \\ China
}

Received: March 10, 2021. Revised: July 13, 2021. Accepted: August 8, 2021. Published: August 11, 2021.

\begin{abstract}
In order to improve the segmentation accuracy of brain tumor magnetic resonance medical image, a segmentation method of brain tumor magnetic resonance medical image based on multi-scale color wavelet texture features is proposed. The segmentation model of brain tumor magnetic resonance medical image is established, and the motion damage information of brain tumor magnetic resonance medical image is adaptively fused in the ultrasound imaging environment. The medical image information is enhanced by using the motion skeletal muscle block matching technology. According to the suspicious point feature matching method of brain tumor, the fusion detection and processing of brain tumor magnetic resonance medical image are carried out. The multi-scale color wavelet texture feature detection method is used to extract the image features of brain tumor MRI points, and the CT bright spot features are used to analyze the features of brain tumor MRI medical images. Combined with the adaptive neural network training method, the automatic detection of brain tumor magnetic resonance medical image is completed, and the suspected brain tumor points are extracted, so as to realize the segmentation of brain tumor magnetic resonance medical image. Simulation results show that the proposed method can effectively improve the segmentation accuracy of brain tumor MRI medical image, and has high resolution and accuracy for suspicious brain tumor detection.
\end{abstract}

Keywords-Brain tumor, characteristics, multi-scale color, magnetic resonance, medical images, segmentation, wavelet texture.

\section{INTRODUCTION}

$\mathrm{W}$ ITH the development of medical imaging technology, the way of understanding brain structure and treatment through anatomy in the early stage has been replaced by imaging technology. Medical imaging technology mainly includes CT scanning, ultrasound and Magneti Resonance Images (MRI), etc. [1]. These collected medical images are used to reconstruct three-dimensional images of internal organs of human body to assist doctors in disease diagnosis and treatment. Among them, MRI has become the most commonly used medical image in the diagnosis and treatment of brain tumors due to its high safety (no radioactive radiation) and high resolution of human soft tissue imaging [2]. In order to diagnose and treat brain tumors, doctors first need to detect and segment the tumors. However, manual positioning and segmentation takes a lot of time.

Yang et al. put forward the application of CT segmentation method in medical images, using computer technology to automatically segment brain tumor medical images, which reduces the requirements of medical image segmentation for doctors' professional skills and reduces doctors' work pressure. Although the tumor region can be separated from the image, the accuracy of image segmentation is not high enough [3]. Kadyrov et al. proposed the application method of wavelet analysis in X-ray CT histogram phase segmentation. According to the similarity of image regions and the difference between regions, the tumor region image is automatically segmented. Using the core technology of image feature extraction, the lesion region is accurately segmented and the work efficiency is improved. However, the resolution of brain tumor detection is poor, which affects the analysis results [4]. Preethi et al. proposed support vector machine (SVM) and Otsu method for the analysis and research of spinal imaging tissue. Fuzzy feature analysis method is used to detect and recognize brain tumor magnetic resonance medical images. Information fusion can improve the recognition ability of brain tumor magnetic resonance medical images. But there are still problems of low detection resolution and accuracy [5].

In order to solve the shortcomings of existing methods, this paper proposes a segmentation method of brain tumor magnetic resonance medical image based on multi-scale color wavelet 
texture features. The main contents of this paper are as follows:

(1) A brain tumor magnetic resonance medical image segmentation model is established.

(2) CT bright spot features are used for transmission analysis of brain tumor magnetic resonance medical image feature details, and an adaptive neural network training method is adopted for automatic detection of brain tumor magnetic resonance medical images to extract suspected brain tumor points.

(3) Realizing brain tumor magnetic resonance medical image segmentation.

(4) Finally, the simulation test analysis is carried out and the validity conclusion is drawn.

Through the research of this paper, it is proved that the segmentation method of brain tumor MRI medical image based on multi-scale color wavelet texture features can help doctors to provide more intuitive and accurate results, which is of great significance to medical image analysis.

\section{BASIC DEFINITIONS}

\section{A. Magnetic resonance medical image sampling of brain} tumors

In order to realize brain tumor magnetic resonance medical image segmentation based on multi-scale color wavelet texture feature extraction, a brain tumor magnetic resonance medical image feature extraction and information sampling model is established, information of brain tumor magnetic resonance medical image is adopted by combining a high-resolution CT scanning method, brain tumor magnetic resonance medical image detection is carried out in an ultrasonic imaging environment, and image information fusion is carried out by combining brain tumor feature distribution [6], and the high-resolution information sampling formula of the image is as follows:

$\left\{\begin{array}{c}\dot{x}(t)=-A x(t)+W f(y(t-\sigma)) \\ \dot{y}(t)=-C y(t)+D x(t-\tau)\end{array}\right.$

A single frame vector feature decomposition method is adopted to segment magnetic resonance medical images of brain tumors, and a kernel function $k_{z}^{g}(t, \tau)$ for image detection is obtained as follows:

$$
\begin{aligned}
& x(t)=\left[x_{1}(t), x_{2}(t), \ldots, x_{n}(t)\right]^{T} \\
& y(t)=\left[y_{1}(t), y_{2}(t), \ldots, y_{n}(t)\right]^{T} \\
& f(y(t))=\left[f_{1}\left(y_{1}(t)\right), f_{2}\left(y_{2}(t)\right), \ldots, f_{n}\left(y_{n}(t)\right]^{T}\right.
\end{aligned}
$$

Harris corner detection technology is adopted to detect corners of magnetic resonance medical images of brain tumors, and the following results are obtained:

$$
f(y(t))=g\left(y(t)+p^{*}\right)-g\left(p^{*}\right)
$$

The frame information of the brain tumor magnetic resonance medical image is recorded as $W_{z}^{g}(n, m)$, and the pixel characteristic distribution of the obtained brain tumor magnetic resonance medical image is as follows: $k_{z}^{g}(t, \tau)=k_{z}(t, \tau)=z\left(t+\frac{\tau}{2}\right) z^{*}\left(t-\frac{\tau}{2}\right)$

The output stability of magnetic resonance medical images of brain tumors is adjusted by adopting a feedback adjustment method, and the image edge contour feature quantity meets the following requirements:

$$
E\left[\mathbf{V}(k) \mathbf{V}^{T}(k)\right]=\left[\begin{array}{cccc}
\mathbf{R}_{v}^{1}(k) & \mathbf{D}_{12}(k) & \cdots & \mathbf{D}_{1 N}(k) \\
\mathbf{D}_{21}(k) & \mathbf{R}_{v}^{2}(k) & \cdots & \mathbf{D}_{2 N}(k) \\
\vdots & \vdots & \ddots & \vdots \\
\mathbf{D}_{N 1}(k) & \mathbf{D}_{N 2}(k) & \cdots & \mathbf{R}_{v}^{N}(k)
\end{array}\right]:=\mathbf{R}_{\mathbf{V}}(k)
$$

Based on super-resolution information feature recognition method, edge contour feature detection and information fusion of brain tumor magnetic resonance medical images are carried out [7]-[9].

\section{B. Magnetic resonance medical image fusion of brain} tumors

Brain tumor information adaptive fusion of brain tumor magnetic resonance medical images is carried out in an ultrasonic imaging environment [10] [11]. brain tumor magnetic resonance information block matching technology is adopted to carry out information enhancement processing of brain tumor magnetic resonance medical images, and the weighting function of motion block matching feature quantity $\mathbf{w}(k)$ and $\mathbf{w}(k)$ are as follows:

$$
E\left[\mathbf{w}(k) \mathbf{V}^{T}(k)\right]=\left[\begin{array}{llll}
\mathbf{B}_{1}(k) & \mathbf{B}_{2}(k) & \cdots & \mathbf{B}_{N}(k)
\end{array}\right]:=\mathbf{B}(k)
$$

Among them, $c(k)=\operatorname{tr}[\mathbf{N}(k)] / \operatorname{tr}[\mathbf{C}(k)]$, the distribution of edge contour corners of brain tumor magnetic resonance medical images is:

$$
\left\{\begin{array}{l}
\mathbf{N}(k)=\mathbf{V}_{0}(k)-\beta \mathbf{R}(k)-\breve{\mathbf{H}}(k) \breve{\mathbf{Q}}(k-1) \breve{\mathbf{H}}^{T}(k) \\
\mathbf{C}(k)=\mathbf{\Phi}(k-1) \mathbf{P}(k-1 \mid k-1) \boldsymbol{\Phi}^{T}(k-1) \breve{\mathbf{H}}^{T}(k) \breve{\mathbf{H}}(k)
\end{array}\right.
$$

The two-dimensional feature quantity of magnetic resonance medical image of brain tumor obtained by fuzzy information feature segmentation method is $\mathbf{R}_{V}(k)$, which is expressed as:

$\mathbf{R}_{V}(k)=\mathbf{L}(k) \mathbf{R}(k) \mathbf{L}^{T}(k)$

where, $\mathbf{R}(k)=\operatorname{diag}\left\{r_{1}(k), r_{2}(k) \ldots, r_{N q}(k)\right\}$ is the pixel intensity of brain tumor magnetic resonance medical images, and the fuzzy statistical feature quantity is S, fuzzy pixel feature extraction is carried out on brain tumor magnetic resonance medical images to obtain image enhancement results as follows: $\dot{V}_{1}(x(t)) \leq 2 x^{T}(t) P A x(t)+2 x^{T}(t) P B x(t-d(t))-(1-\tau) x^{T}(t-d(t)) Q_{2} x(t-d(t))$

$$
+x^{T}(t) Q_{1} x(t)-\left(1-\tau_{1}\right) x^{T}\left(t-d_{1}(t)\right)\left(Q_{1}-Q_{2}\right) x\left(t-d_{1}(t)\right),
$$

$\dot{V}_{2}(x(t))=x^{T}(t)\left(R_{1}+R_{2}\right) x(t)-x^{T}(t-h) R_{1} x(t-h)-x^{T}\left(t-h_{1}\right) R_{2} x\left(t-h_{1}\right)$,

$\dot{V}_{3}(x(t))=\dot{x}^{T}(t)\left(h_{1} Z_{1}+h_{2} Z_{2}+h Z_{3}\right) \dot{x}(t)-\int_{t-h_{1}}^{t} \dot{x}^{T}(s) Z_{1} \dot{x}(s) d s$

$$
-\int_{t-h}^{t-h_{1}} \dot{x}^{T}(s) Z_{2} \dot{x}(s) d s-\int_{t-h}^{t} \dot{x}^{T}(s) Z_{3} \dot{x}(s) d s .
$$

Carrying out fusion processing on brightness components to obtain an information fusion matrix 
$K\left(Z_{1}+Z_{2}+Z_{3}\right)^{-1} K^{T}, W Z_{1}^{-1} W^{T} \quad$ and $\quad L\left(Z_{2}+Z_{3}\right)^{-1} L^{T} \quad$ in $0 \leq d_{1}(t) \leq h_{1}$; In a low-illumination image, the brain tumor feature quantity is $\Psi\left(d_{1}(t), d_{2}(t)\right)<0$ if and only if:

$$
\begin{aligned}
& \Psi\left(h_{1}, h_{2}\right)=\Psi+h_{1} K\left(Z_{1}+Z_{2}+Z_{3}\right)^{-1} K^{T}+h_{2} M\left(Z_{2}+Z_{3}\right)^{-1} M^{T}<0, \\
& \Psi\left(h_{1}, 0\right)=\Psi+h_{1} K\left(Z_{1}+Z_{2}+Z_{3}\right)^{-1} K^{T}+h_{2} L\left(Z_{2}+Z_{3}\right)^{-1} L^{T}<0, \\
& \Psi\left(0, h_{2}\right)=\Psi+h_{1} W Z_{1}^{-1} W^{T}+h_{1} L\left(Z_{2}+Z_{3}\right)^{-1} L^{T}+h_{2} M\left(Z_{2}+Z_{3}\right)^{-1} M^{T}<0, \\
& \Psi(0,0)=\Psi+h_{1} W Z_{1}^{-1} W^{T}+h_{1} L\left(Z_{2}+Z_{3}\right)^{-1} L^{T}+h_{2} L\left(Z_{2}+Z_{3}\right)^{-1} L^{T}<0 .
\end{aligned}
$$

Brain tumor suspected point feature matching method is used for fusion detection processing of brain tumor magnetic resonance medical images, and brain tumor magnetic resonance point multi-scale color wavelet texture feature detection method is used for image feature extraction to improve image fusion capability [12] [13].

\section{Magnetic Resonance Medical Image Segmentation OF BRAIN TUMORS}

\section{A. Color and texture characteristics of color images}

The color features of the image should be analyzed before multi-scale color wavelet texture detection of brain tumor. The multi-scale color image of brain tumor collected by imaging system is generally RGB image. The RGB image is suitable for the display of the image, but the R, G, B color composition has a high correlation and the RGB chromaticity space does not represent the real color difference, and it is not suitable for color feature extraction. In image processing, RGB images are generally transformed into other chromaticity spaces, such as YUV, HIS, Lab, etc., each chromaticity space has its own advantages and disadvantages. In the $\mathrm{YCbCr}$ chromaticity space, the correlation between its color components is small and the image features can be effectively represented. Therefore, the following image color features of the $\mathrm{YCbCr}$ chromaticity space are studied, as shown in Fig. 1. The specific expression of RGB chromaticity space converted into $\mathrm{YCbCr}$ chromaticity space is:

$\left[\begin{array}{l}Y \\ C_{b} \\ C_{r}\end{array}\right]=\left[\begin{array}{ccc}65.481 & 128.553 & 24.966 \\ -39.793 & -74.203 & 112.000 \\ 112.000 & -93.786 & -18.214\end{array}\right] \times\left[\begin{array}{c}R \\ G \\ B\end{array}\right]+\left[\begin{array}{c}16 \\ 128 \\ 128\end{array}\right]$

$$
\begin{aligned}
& I_{x y}^{L}=\frac{1}{N_{l}} \sum_{i=0}^{N_{l}-1} l(i) I_{[(x+i-1) \bmod M](y-1)} \\
& I_{x y}^{H}=\frac{1}{N_{h}} \sum_{j=0}^{N_{h}-1} h(i) I_{[(x+j-1) \bmod M](y-1)}
\end{aligned}
$$

where $\mathrm{x}=0,2,4, \ldots, \mathrm{M}, \mathrm{y}=0,1,2, \ldots, \mathrm{N}$. Filter again to get:

$$
\begin{aligned}
& I_{x y}^{L H}=\frac{1}{N_{h}} \sum_{j=0}^{N_{h}-1} h(i) I_{(x / 2-1)[(y+i-1) \bmod N]}^{L} \\
& I_{x y}^{H L}=\frac{1}{N_{l}} \sum_{i=0}^{N_{l}-1} l(i) I_{(x / 2-1)[(y+i-1) \bmod N]}^{H}
\end{aligned}
$$




$$
I_{x y}^{H H}=\frac{1}{N_{h}} \sum_{j=0}^{N_{h}-1} h(i) I_{(x / 2-1)[(y+i-1) \bmod N]}^{H}
$$

where $\mathrm{x}=0,2,4, \ldots, \mathrm{M}, \mathrm{y}=0,2,4, \ldots, \mathrm{N}$. To some extent, the selection of wave filters will affect the time used to extract and segment texture features. The Daubechies Wavelet has orthogonality, tightness and low complexity, and its speed is fast. Therefore, in order to ensure the computational speed and the validity of extracting features, the $\mathrm{db} 1$ wavelet is selected in image segmentation. The wavelet decomposition subgraph corresponding to the Fig. 1 image is shown in Fig. 2.

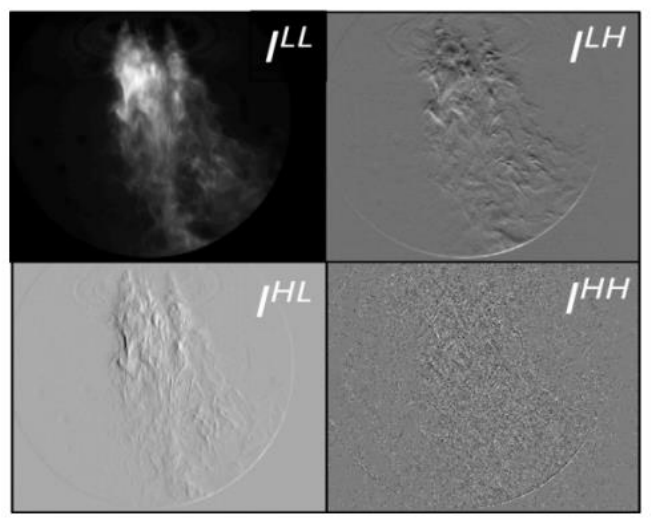

Fig. 2 wavelet decomposition of image corresponding to Fig. 1

The wavelet detail image is the energy distribution of each channel of the original image, which contains the texture information in different directions. It can be seen that after the wavelet segmentation, the image texture becomes clear and the imaging is obvious.

So the energy of the detail subgraph is extracted as the texture feature, that is:

$T_{x y}^{f}=\frac{1}{(2 v+1)^{2}} I^{f}$

where, $f$ is the selection window width, that is, the pixel block width selected for each calculation of the energy. The feature vector of each pixel of the image is its corresponding color feature and the wavelet texture eigenvalue of each angle, then the feature vector corresponding to the pixel point $(x, y)$ of the image can be characterized as:

$\mathbf{F}=\left[\begin{array}{cccc}T_{11}^{f} & T_{12}^{f} & \ldots & T_{1 y}^{f} \\ T_{21}^{f} & T_{22}^{f} & \ldots & T_{2 y}^{f} \\ \ldots & \ldots & \ldots & \ldots \\ T_{x 1}^{f} & T_{x 2}^{f} & \ldots & T_{x y}^{f}\end{array}\right]$

Based on the above contents, the color and texture features of color images are analyzed, and the multi-scale color wavelet texture features of brain tumors are detected.

B. Multi-scale color wavelet texture feature detection of magnetic resonance points in brain tumors

In order to improve the computing speed, a medical image segmentation algorithm based on multi-scale color wavelet texture features is proposed. Firstly, the image feature matrix is compressed. In order to eliminate the influence of noise and preserve the local feature, the local mean method is used to compress the feature matrix. Then, the compression scale region is obtained and the edges are detected by the classification algorithm. Finally, according to the compression scale edge further detects it in the original scale region, constructs the edge region characteristic matrix and further detects, thus obtains the original scale region image.

Using the bright region weight analysis method, multi-dimensional scale decomposition of magnetic resonance medical images of brain tumors is carried out, and the nuclear spatial feature distribution set is obtained as follows:

$$
\begin{aligned}
{\left[\mathbf{I}+\mathbf{P} \mathbf{H}^{\mathrm{T}} \mathbf{R}^{-1} \mathbf{H}\right]^{-1} \mathbf{P} } & =\left[\mathbf{P}^{-1}+\mathbf{H}^{\mathrm{T}} \mathbf{R}^{-1} \mathbf{H}\right]^{-1} \\
& =\mathbf{P}-\mathbf{P} \mathbf{H}^{\mathrm{T}}\left(\mathbf{H} \mathbf{P} \mathbf{H}^{\mathrm{T}}+\mathbf{R}\right)^{-1} \mathbf{H P}
\end{aligned}
$$

Using the bright region weight analysis method, multi-dimensional scale decomposition of magnetic resonance medical images of brain tumors is carried out [12], and the nuclear spatial feature distribution set is obtained as follows:

$$
\begin{aligned}
\hat{\mathbf{y}} & (k \mid k-1)=\mathbf{Y}(k \mid k-1) \hat{\mathbf{x}}(k \mid k-1) \\
& =\left\{\mathbf{I}-\mathbf{F}(k-1)\left[\mathbf{F}(k-1)+\mathbf{Q}^{-1}(k-1)\right]^{-1}\right\} \\
& \times \mathbf{F}(k-1)[\mathbf{\Phi}(k-1) \hat{\mathbf{x}}(k-1 \mid k-1)+\mathbf{J}(k-1) \breve{M}(k-1)] \\
& =\left\{\mathbf{I}-\mathbf{F}(k-1)\left[\mathbf{F}(k-1)+\mathbf{Q}^{-1}(k-1)\right]^{-1}\right\} \lambda^{-1}(k) \\
& \times\left[\Phi^{-1}(k-1)\right]^{\mathrm{T}} \mathbf{Y}(k-1 \mid k-1)[\hat{\mathbf{x}}(k-1 \mid k-1) \\
& \left.+\boldsymbol{\Phi}^{-1}(k-1) \mathbf{J}(k-1) \mathbf{M}(k-1)\right] \\
& =\lambda^{-1}(k)\left\{\mathbf{I}-\mathbf{F}(k-1)\left[\mathbf{F}(k-1)+\mathbf{Q}^{-1}(k-1)\right]^{-1}\right\} \\
& \times\left[\Phi^{-1}(k-1)\right]^{\mathrm{T}}[\hat{\mathbf{y}}(k-1 \mid k-1)+\hat{\mathbf{i}}(k-1)]
\end{aligned}
$$

wherein, $\quad \hat{\mathbf{i}}(k-1)=\mathbf{Y}(k-1 \mid k-1) \mathbf{\Phi}^{-1}(k-1) \mathbf{J}(k-1) \breve{\mathbf{M}}(k-1)$.

Base on that feature fusion scheduling method, the obtain quantitative feature prediction values of the ultrasonic brain tumor magnetic resonance medical images of the brain tumor are as follows:

$$
\begin{aligned}
\mathbf{K}(k) & =\mathbf{P}(k \mid k-1) \breve{\mathbf{H}}^{T}(k)\left[\breve{\mathbf{H}}(k) \mathbf{P}(k \mid k-1) \breve{\mathbf{H}}^{T}(k)+\mathbf{R}(k)\right]^{-1} \\
= & \mathbf{P}(k \mid k-1) \breve{\mathbf{H}}^{T}(k)\left\{\left[\breve{\mathbf{H}}(k) \mathbf{P}(k \mid k-1) \breve{\mathbf{H}}^{T}(k)+\mathbf{R}(k)\right]^{-1}\right. \\
& \left.+\mathbf{R}^{-1}(k)-\mathbf{R}^{-1}(k)\right\} \\
= & \mathbf{P}(k \mid k-1) \breve{\mathbf{H}}^{T}(k) \mathbf{R}^{-1}(k)+\mathbf{P}(k \mid k-1) \breve{\mathbf{H}}^{T}(k) \\
& \times\left[\breve{\mathbf{H}}(k) \mathbf{P}(k \mid k-1) \breve{\mathbf{H}}^{T}(k)+\mathbf{R}(k)\right]^{-1} \\
& \times\left\{\mathbf{I}-\left[\breve{\mathbf{H}}(k) \mathbf{P}(k \mid k-1) \breve{\mathbf{H}}^{T}(k)+\mathbf{R}(k)\right] \mathbf{R}^{-1}(k)\right\} \\
= & \left\{\mathbf{P}(k \mid k-1)+\mathbf{P}(k \mid k-1) \breve{\mathbf{H}}^{T}(k)\right. \\
& \times\left[\breve{\mathbf{H}}(k) \mathbf{P}(k \mid k-1) \breve{\mathbf{H}}^{T}(k)+\mathbf{R}(k)\right]^{-1} \\
& \times \breve{\mathbf{H}}(k) \mathbf{P}(k \mid k-1)\} \breve{\mathbf{H}}^{T}(k) \mathbf{R}^{-1}(k) \\
= & \mathbf{P}(k \mid k) \breve{\mathbf{H}}^{T}(k) \mathbf{R}^{-1}(k)
\end{aligned}
$$

By introducing the segmented target and background image fusion matrix, the boundary feature information distribution of ultrasound medicine for brain tumors can be obtained as follows:

$\left\{\begin{array}{l}\mathbf{F}(k-1)=\lambda^{-1}(k)\left[\boldsymbol{\Phi}^{-1}(k-1)\right]^{\mathrm{T}} \mathbf{Y}(k-1 \mid k-1) \boldsymbol{\Phi}^{-1}(k-1) \\ \hat{\mathbf{i}}(k-1)=\mathbf{Y}(k-1 \mid k-1) \mathbf{\Phi}^{-1}(k-1) \mathbf{J}(k-1) \breve{\mathbf{M}}(k-1)\end{array}\right.$

The dual relationship between the target and the background image can be expressed as: 
$\min F=R^{2}+A \sum_{i} \xi_{i}$

s.t : $\left\|\phi\left(x_{i}\right)-o\right\|^{2} \leq R^{2}+\xi_{i}$ and $\xi_{i} \geq 0, i=1,2, \ldots$

$\max \sum_{i} \alpha_{i} K\left(x_{i}, x_{i}\right)-\sum_{i} \sum_{j} \alpha_{i} \alpha_{j} K\left(x_{i}, x_{j}\right)$

s.t $: \sum_{i} \alpha_{i}=1$ and $0 \leq \alpha_{i} \leq A, i=1,2, \ldots$

Then, $\sum_{i} \alpha_{i}=1, K\left(x_{i}, x_{i}\right)=1$, feature matching method of

suspected brain tumor points is used for fusion detection of brain tumor magnetic resonance medical images, and multi-scale color wavelet texture feature detection method of brain tumor magnetic resonance points is used for image feature extraction [13] [15].

\section{Segmentation of MRI medical images of brain tumors by} ultrasound

An adaptive neural network training method is adopted to automatically detect brain tumor magnetic resonance medical images on the extracted brain tumor suspected points [16], and a convolution neural network structural model for brain tumor magnetic resonance medical image segmentation is as follows:

$$
\begin{aligned}
& \frac{\partial L}{\partial R}=0 \rightarrow \sum_{i} \alpha_{i}=1 \\
& \frac{\partial L}{\partial o}=0 \rightarrow \mathrm{o}=\frac{\sum_{i} \alpha_{i} x_{i}}{\sum_{i} \alpha_{i}}=\sum_{i} \alpha_{i} x_{i} \\
& \frac{\partial L}{\partial \xi_{i}}=0 \rightarrow \mathrm{A}-\alpha_{i}-\gamma_{i}=0
\end{aligned}
$$

Obtaining the detail texture of the magnetic resonance image of the source brain tumor, satisfying $\mathrm{A}-\alpha_{i}-\gamma_{i}=0$, including:

$d^{2}\left(x_{i}\right)=\left(x_{i}, x_{i}\right)-2 \sum_{j} \alpha_{i}\left(x_{j}, x_{i}\right)+\sum_{i} \sum_{l} \alpha_{j} \alpha_{l}\left(x_{j}, x_{l}\right)$

According to the quality of brain tumor magnetic resonance image fusion, brain tumor magnetic resonance medical image segmentation is performed, and the threshold segmentation function is obtained as follows:

$\max 1-\sum_{i} \sum_{j} \alpha_{i} \alpha_{j} K\left(x_{i}, x_{j}\right)$
s.t $: \sum_{i} \alpha_{i}=1$ and $0 \leq \alpha_{i} \leq A, i=1,2, \ldots$

wherein, $K\left(x_{i}, x_{j}\right)=e^{\frac{-\left\|x_{i}-x_{j}\right\|^{2}}{2 \sigma^{2}}}$, according to the inter-class variance of brain tumor magnetic resonance medical image, the optimal segmentation is carried out, and the optimal threshold value calculation of brain tumor magnetic resonance medical image segmentation is obtained:

$$
\begin{aligned}
& \hat{\mathbf{x}}(k \mid k-1)=\mathbf{\Phi}(k-1) \hat{\mathbf{x}}(k-1 \mid k-1)+\mathbf{J}(k-1) \breve{\mathbf{M}}(k-1) \\
& \mathbf{P}(k \mid k-1)=\lambda(k) \mathbf{\Phi}(k-1) \mathbf{P}(k-1 \mid k-1) \mathbf{\Phi}^{T}(k-1)+\breve{\mathbf{Q}}(k-1) \\
& \mathbf{K}(k)=\mathbf{P}(k \mid k-1) \breve{\mathbf{H}}^{T}(k)\left[\breve{\mathbf{H}}(k) \mathbf{P}(k \mid k-1) \breve{\mathbf{H}}^{T}(k)+\mathbf{R}(k)\right]^{-1} \\
& \hat{\mathbf{x}}(k \mid k)=\hat{\mathbf{x}}(k \mid k-1)+\mathbf{K}(k)[\breve{\mathbf{M}}(k)-\breve{\mathbf{H}}(k) \hat{\mathbf{x}}(k \mid k-1)] \\
& \mathbf{P}(k \mid k)=[\mathbf{I}-\mathbf{K}(k) \breve{\mathbf{H}}(k)] \mathbf{P}(k \mid k-1)
\end{aligned}
$$

where I represents the N-order covariance identity matrix. To sum up, CT bright spot features are used to carry out transmission analysis of features of brain tumor magnetic resonance medical images to realize segmentation of brain tumor magnetic resonance medical images [17].

\section{Simulation Test Analysis}

In order to prove the effectiveness of the proposed method based on multi-scale color wavelet texture features for brain tumor MRI medical image segmentation, the image segmentation test is carried out through MATLAB simulation platform. By comparing with $\mathrm{CT}$ segmentation, wavelet segmentation and Otsu method, it is proved that the image segmentation accuracy of this method is high, and the detection resolution and accuracy of suspicious brain tumors are high.

\section{A. Simulation experiment platform and parameter setting}

MATLAB 7.0 is used to design a simulation tool for brain tumor magnetic resonance medical image segmentation. Where the simulation platform interface for image segmentation is shown in Fig. 3.

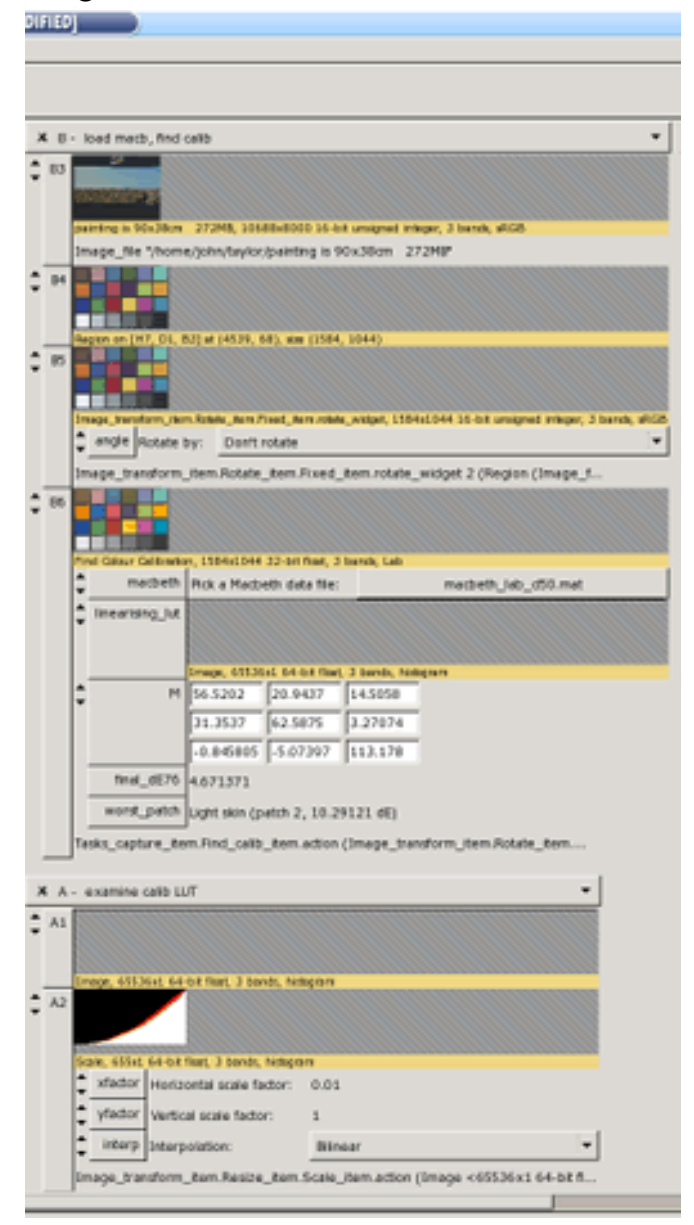

Fig. 3 image processing simulation interface

\section{B. Experiment and analysis of brain tumor MRI medical image segmentation}

The low frequency coefficient for ultrasound brain tumor magnetic resonance medical image fusion is 0.25 , the scale of initial segmentation is 12 , the low frequency sub-band coefficient is 0.85 , and the quality index parameter of image 
segmentation is 0.57. According to the above simulation parameter settings, brain tumor magnetic resonance medical image segmentation is performed. The original sample is shown in Fig. 4. The extracted tumor image is shown in Fig. 5.

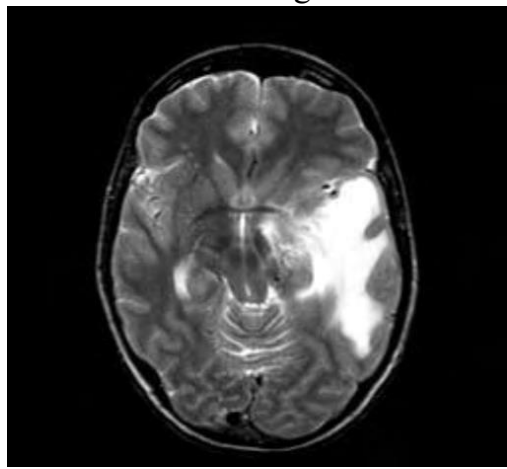

Fig. 4 MRI image of brain

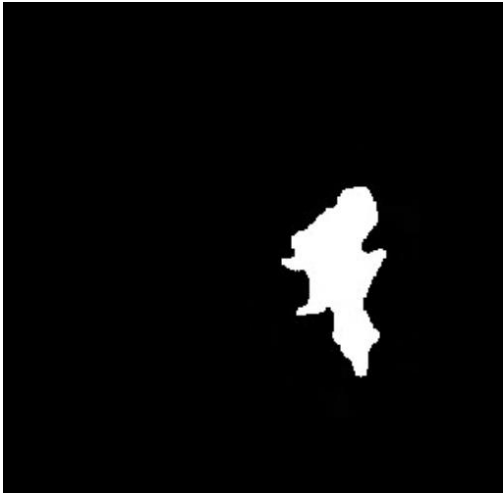

Fig. 5 extracted tumor image

Taking the images of Fig. 4 and Fig. 5 as the research object, magnetic resonance medical image segmentation of brain tumor is performed, and the segmentation result is shown in Fig. 6.

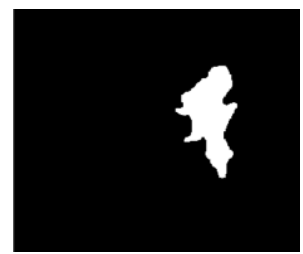

(a) Image segmentation output

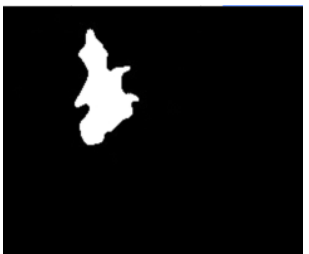

(b) Image after 180 degree rotation
Fig. 6 segmentation results of magnetic resonance medical images of brain tumors

Fig. 6 shows that the method in this paper can effectively realize brain tumor magnetic resonance medical image segmentation with high segmentation accuracy, and test the error of brain tumor magnetic resonance medical image segmentation output. the comparison results are shown in Table I.

Table I Error comparison of magnetic resonance medical image segmentation of brain tumors

\begin{tabular}{ccccc}
\hline $\begin{array}{c}\text { Number } \\
\text { of } \\
\text { iterations }\end{array}$ & $\begin{array}{c}\text { This } \\
\text { method }\end{array}$ & $\begin{array}{c}\text { CT } \\
\text { segmentation }\end{array}$ & $\begin{array}{c}\text { Wavelet } \\
\text { segmentation }\end{array}$ & $\begin{array}{c}\text { Otsu } \\
\text { method }\end{array}$ \\
\hline 100 & 0.134 & 0.356 & 0.295 & 0.452 \\
200 & 0.056 & 0.278 & 0.267 & 0.376 \\
300 & 0.034 & 0.154 & 0.122 & 0.403 \\
400 & 0.014 & 0.143 & 0.114 & 0.393 \\
500 & 0 & 0.113 & 0.055 & 0.362 \\
\hline
\end{tabular}

Table I shows that the error of brain tumor magnetic resonance medical image segmentation using the method in this paper is low.

C. Comparative analysis of segmentation performance of brain tumor magnetic resonance medical image

In order to further test the effectiveness and feasibility of this method, the performance of the segmentation algorithm will be analyzed. The experiments were performed using PRI (probabilistic rand index), VOI (visualization information), GCE (global constancy error), and BDE (boundary displacement error) to analyze and evaluate the segmentation results from different angles. PRI is the ratio of pixels to the total number of pixels that are consistent between machine and manual segmentation; VOI is used to measure the distance between two segmented images, represents the randomness of segmentation image relative to artificial segmentation. GCE is used to measure the consistency of segmented images relative to manually segmented images. BDE measures the edge average error of the two segmentation images, defined as the distance between a pixel on the edge of the automatic segmentation and the nearest pixel on the artificial segmentation image. The larger the PRI value in the quantitative results, the smaller the VOI, GCE, and BDE values, indicating that the closer the unsupervised segmentation result is to the manual segmentation result, the better the segmentation effect is. And the results are shown in Table II.

Table II Performance analysis of segmentation algorithm

\begin{tabular}{ccccccccccccc}
\hline $\begin{array}{c}\text { Performance } \\
\text { analysis }\end{array}$ & \multicolumn{3}{c}{ This method } & \multicolumn{3}{c}{ CT segmentation } & \multicolumn{2}{c}{ Wavelet segmentation } & \multicolumn{3}{c}{ Otsu method } \\
\hline Bear fruit & $t_{1}$ & $t_{2}$ & $T_{a}$ & $t_{1}$ & $t_{2}$ & $T_{a}$ & $t_{1}$ & $t_{2}$ & $T_{a}$ & $t_{1}$ & $t_{2}$ & $T_{a}$ \\
Fractional error & 0 & 0 & 0 & 0.09 & 0.06 & 0.01 & 0.12 & 0.20 & 0.18 & 0.11 & 0.13 & 0.10 \\
Absolute error & 0 & 0 & 0 & -0.4 & -0.2 & 0.1 & 0.2 & 0.1 & 0.4 & 3.4 & 2.2 & 4.1 \\
Average value & 60.2 & 56.2 & 57.7 & 61 & 123 & 104 & 64.3 & 64.0 & 100 & 103 & 132 & 193 \\
Time consuming & & 432.45 & & & 562.43 & & & 552.14 & & & 1734.13 \\
t/s & & & & & & & & & & & &
\end{tabular}

In the table, $t_{1}, t_{2}$ is threshold, $T_{a}$ is Maximum entropy.

Table II shows that the error value of this method is low and time consuming less.

In order to quantify the segmentation results, different medical images were randomly selected and segmented by Otsu,
CT segmentation, Wavelet segmentation and this paper, Calculate the mean and standard deviation of PRI, VOI, GCE and BDE for each segmentation method and the time required to segment the flame image, thus evaluating the performance of the segmentation algorithm from an objective point of view. Fig. 7 
shows the quantitative evaluation index mean and standard deviation of the segmentation results of different segmentation

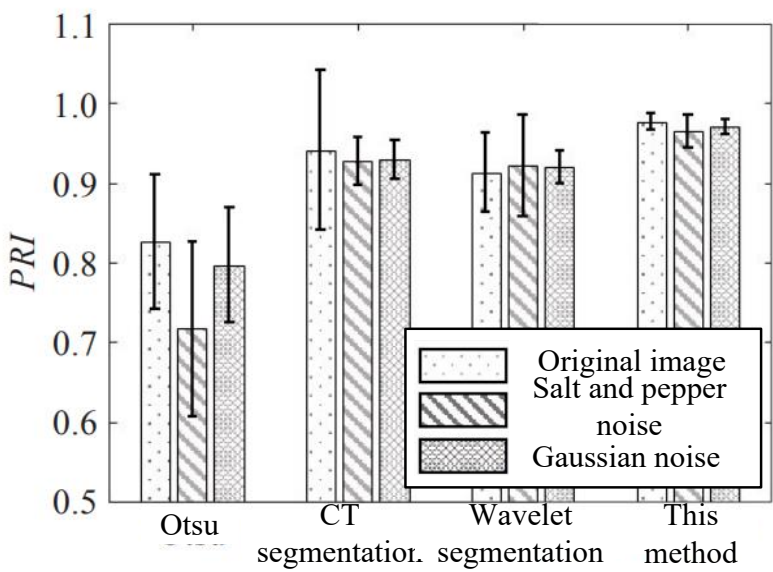

(a) PRI

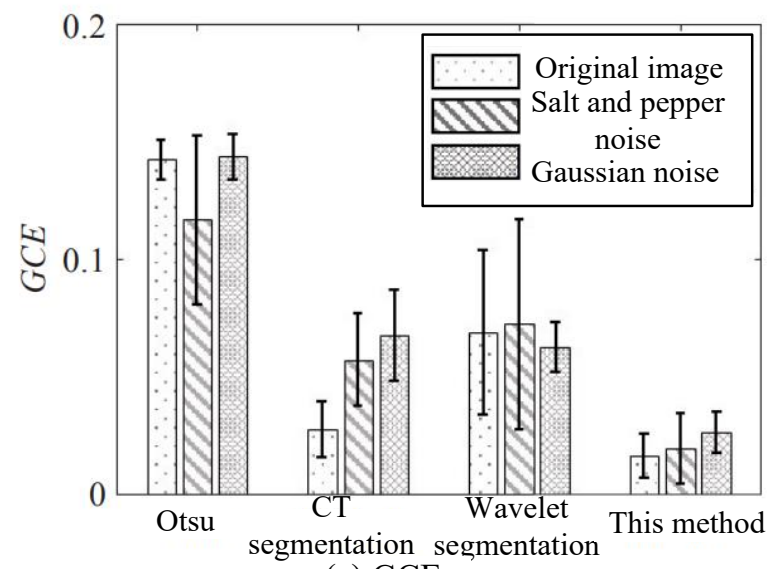

(c) GCE methods.

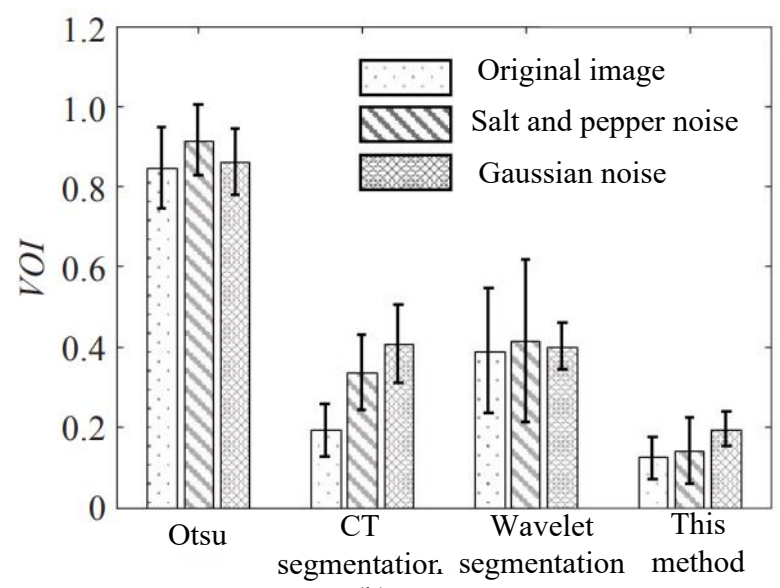

(b) VOI

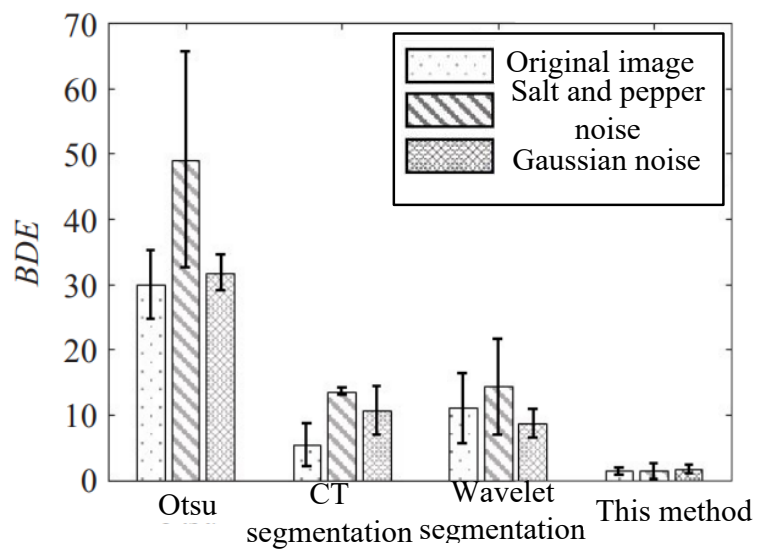

(d) BDE

Fig. 7 evaluation index of image segmentation algorithm

As shown in Fig. 7, for the original medical image, the evaluation indexes of Otsu and CT segmentation methods are obviously affected by salt and pepper noise and Gaussian noise, and the detection resolution and accuracy are low; Although the influence of wavelet segmentation method on noise is less than that of Otsu and CT segmentation methods, the segmentation evaluation result is not ideal; The proposed method is superior to the other three methods. It has the least influence after adding salt and pepper noise and Gaussian noise, and always keeps good segmentation results. It has high resolution and accuracy for suspicious brain tumor detection.

\section{CONCLUSION}

In order to solve the problems of low segmentation accuracy, low resolution and accuracy for suspected brain tumor detection, this paper proposes a segmentation method of brain tumor magnetic resonance medical image based on multi-scale color wavelet texture features. The segmentation model of brain tumor magnetic resonance medical image is established. The feature details of medical image are analyzed by CT highlight features. The adaptive neural network training method is used to automatically detect the medical image and extract suspicious brain tumor points. Compared with the existing methods, it can be seen that the segmentation accuracy of the proposed method is higher, and the detection resolution and accuracy are still higher than the other three methods after being affected by noise, which proves the effectiveness of the proposed method and has important significance for the analysis of actual brain tumor MRI medical images.

Due to the limited time, this paper only analyzes the medical image segmentation. In the following research, we can focus on the image texture to study the directionality, symmetry and regularity of the texture in the real medical image. According to the characteristics of the image texture, we choose the technology of wavelet transform which is more matching with the image texture, which has a broader significance for the research of medical image.

\section{References}

[1] A. Johansson, M. Karlsson, and T. Nyholm, "CT substitute derived from MRI sequences with ultrashort echo time," Medical Physics, vol. 38, no. 5, pp. 2708-2714, 2011. 
[2] A. Johansson, M. Karlsson, and J. Yu, "Voxel-wise uncertainty in CT substitute derived from MRI," Medical Physics, vol. 39, no. 6, pp. 3283-3290, 2012.

[3] X. Yang, Y. Lei, and Y. Liu, "Synthetic MRI-aided Multi-Organ CT segmentation for head and neck radiotherapy treatment planning," International Journal of Radiation Oncology Biology Physics, vol. 108, no. 3, pp. e341, 2020.

[4] R. Kadyrov, A, Starovoytov, and E. Utemov, “Application of wavelet analysis of X-Ray computed tomography histogram for phase segmentation," IOP Conference Series: Earth and Environmental Science, vol. 516, no. 1, pp. 012005 (10pp), 2020.

[5] N. Preethi, C. M. Vinodhini, and D. Nivethaa, "Estimating tissue from backbone using radiograph by SVM and OTSU method," Journal of Physics: Conference Series, vol. 1717, no. 1, pp. 012021 (6pp), 2021.

[6] P. J. Qian, S. W. Sun, and Y. Z. Jiang, "Cross-domain, soft-partition clustering with diversity measure and knowledge reference," Pattern Recognition, vol. 50, pp. 155-177, 2016.

[7] Z. G. Xu, C. Z. Xiao, and Y. B. Liao, "Analysis of the seismic-isolated connected structures for MOMA," China Civil Engineering Journal, vol. 41, no. 3, pp. 53-57, 2008.

[8] Y. Yuan and F. Y. Wang, "Blockchain, the state of the art and future trends," Acta Automatica Sinica, vol. 42, no. 4, pp. 481-494, 2006.

[9] P. He, G. Yu, and Y. F. Zhang, "Survey on blockchain technology and its application prospects," Computer Science, vol. 44, no. 4, pp. 1-7, 15, 2016.

[10] Y. Yuan and F. Y. Wang, "Blockchain, the state of the art and future trends," Acta Automatica Sinica, vol. 42, no. 4, pp. 481-494, 2016.

[11]D. Zhao and X. K. Sun, "Some robust approximate optimality conditions for nonconvex multi-objective optimization problems," Applied Mathematics and Mechanics, vol. 40, no. 6, pp. 694-700, 2019.

[12] G. M. Lee and J. H. Lee, "On nonsmooth optimality theorems for robust multiobjective optimization problems," Journal of Nonlinear and Convex Analysis, vol. 16, no. 10, pp. 2039-2052, 2015.

[13]X. K. Sun, Z. Y. Peng, and X. L. Guo, "Some characterizations of robust optimal solutions for uncertain convex optimization problems," Optimization Letters, vol. 10, no. 7, pp. 1463-1478, 2016.

[14] R. Mamdouh, H. M. El-Bakry, and A. Riad, "Converting 2D-medical image files "DICOM" into 3D- models, based on image processing, and analysing their results with python programming," WSEAS Transactions on Computers, vol. 19, pp. 10-20, 2020.

[15]X. L. Zhong, J. L. Fan, and Y. N. Li, "Multi-light multi-color digital image color difference online detection simulation," Computer Simulation, vol. 36, no. 8, pp. 49-55, 2019.

[16] J. C. Fan and J. Wang, "A two-phase fuzzy clustering algorithm based on neurodynamic optimization with its application for PolSAR image segmentation," IEEE
Transactions on Fuzzy Systems, vol. 26, no. 1, pp. 72-83, 2018.

[17] M. Kiechle, M. Storath, and A. Weinmann, "Model-based learning of local image features for unsupervised texture segmentation," IEEE Transactions on Image Processing, vol. 34, no. 10, pp. 99-104, 2017.

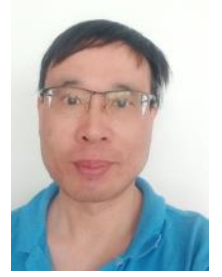

Qi Zhang, male, was born in September, 1972. His title is Master of Communication and Electronic System. He graduated from South East University Information engineering in 1995, major in Information Engineering, with a baccalaureate. He graduated from Xi'an Jiao Tong University in 1998, major in Information engineering, with a master's degree. Now he is working in College of Finance and Information, Ningbo University of Finance and Economics. He mainly engages in the research of Industrial Internet, digital image processing and software engineering. So far, He published more than twenty scientific theses and undertook nine research projects.

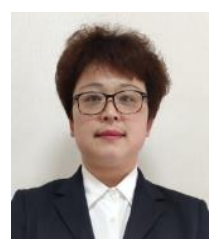

Yan Li, female, was born in August, 1982. Her title is Lecturer. She graduated from Hohai University in 2005, major in computer science, with a baccalaureate. Now she is working in College of Finance and Information, Ningbo University of Finance and Economics. She mainly engages in the research of Software engineering, big data and Internet applications. So far, she published twelve scientific theses and undertook seven research projects. Her interests include software engineering, big data and internet applications.

\section{Author Contribution:}

Qi Zhang: A segmentation method of brain tumor magnetic resonance medical image based on multi-scale color wavelet texture features is proposed.

Yan Li: Combined with the adaptive neural network training method, the automatic detection of brain tumor magnetic resonance medical image is completed.

All authors discussed the results and wrote the manuscript.

\section{Creative Commons Attribution License 4.0 (Attribution 4.0 International, CC BY 4.0)}

This article is published under the terms of the Creative Commons Attribution License 4.0

https://creativecommons.org/licenses/by/4.0/deed.en_US 